\title{
Rare Association of Perivascular Granulomatous Lesions in a Patient with Acute Retinal Necrosis
}

\author{
Takahiro Sogawa Noriyasu Hashida Miki Sawa Kohji Nishida \\ Department of Ophthalmology, Osaka University Graduate School of Medicine, Osaka, \\ Japan
}

\section{Key Words}

Acute retinal necrosis - Optical coherence tomography - Granuloma

\begin{abstract}
Purpose: The aim of this study was to examine sequential changes in perivascular granulomatous lesions with acute retinal necrosis (ARN). Methods: A healthy 46-year-old Japanese woman, who developed floaters and pain in her left eye, underwent optical coherence tomography (OCT), fluorescein angiography, and routine ophthalmological examinations. Treatment-associated changes in perivascular granulomatous lesions were monitored using spectral-domain (SD)-OCT. Results: The patient had no previous ophthalmic history, and her general condition was good. A slit-lamp examination revealed keratic precipitates and aqueous cells $(2+)$ in the left eye. A fundus examination showed yellow-white patches of necrotizing retinal lesions in the temporal upper area, retinal arteritis, retinal hemorrhage, and vitreous opacities. The patient was diagnosed with ARN according to diagnostic criteria. SD-OCT images confirmed high-intensity and uniform granulomatous deposits in the perivascular area and fovea. Systemic corticosteroids and antiviral therapy were initiated, resulting in the gradual resolution of granulomatous lesions. The patient continues to be followed untreated without evidence of recurrence, retinal detachment, or active inflammation. Conclusions: This is the first report of perivascular granulomatous lesions in a patient with ARN. Our results showed that the formation of granulomas may be induced in the retina of ARN patients without fulminant inflammation.

(C) 2015 The Author(s)

Published by S. Karger AG, Basel
\end{abstract}


Sogawa et al.: Rare Association of Perivascular Granulomatous Lesions in a Patient with Acute Retinal Necrosis

\section{Introduction}

Acute retinal necrosis (ARN) is characterized by progressive peripheral necrotizing retinitis, anterior chamber inflammation, retinal arteritis, and vitritis in immunocompetent patients [1]. The clinical course of ARN involves acute iridocyclitis with mutton fat keratic precipitates in the acute phase, retinal vasculitis, and white necrotizing lesions in the peripheral retina. Despite the diagnostic criteria described by the American Uveitis Society in 1994 [2], difficulties are still associated with accurately diagnosing ARN, and its prognosis is poor due to rapidly progressing secondary retinal detachment and optic nerve atrophy. In previous studies, visual outcomes at 6 months were $<6 / 60$ in $48 \%$ of the affected eyes examined [3]. In recent years, detection of the viral genome by conducting a polymerase chain reaction analysis of the aqueous humor is considered useful and important for diagnosing ARN [4]. Although advances in the diagnosis of ARN have recently been achieved, a unified treatment does not currently exist.

Suzuki et al. [5] obtained optical coherence tomography (OCT) images of retinal lesions with ARN; OCT images of the affected lesions of ARN patients showed highly reflective areas in the inner layers of the retina that corresponded with the yellowish-white lesions that are known to occur in the acute phase. The complete absence of a retinal structure was observed, particularly in patients with severe inflammation, in addition to subretinal exudates and/or fluid. In the remission phase, the complete absence of a retinal structure and a significant reduction in retinal thickness were detected on OCT images.

The pathogenesis of ARN is presumed to be associated with herpes virus infection; however, few pathological reports have been published on the eyes of patients with ARN during the active phase. Culbertson et al. [6] performed a histopathological analysis of the eyes of ARN patients in the active phase. All retinal layers became necrotic after viral infection, and retinal necrosis spread to the surrounding healthy retina. A previous study confirmed granulomatous inflammation in the choroid; however, viral antigens were not detected in the nerve fiber layer, retinal pigment epithelium, or choroid in the late phase [6]. Sequential changes in granulomatous lesions with ARN, especially those located in the retina, have not yet been examined because severe inflammation completely disrupts the structure of the whole retina.

In the present study, we report the rare association of perivascular granulomatous lesions in a patient with ARN. We also monitored treatment-associated changes in these lesions by serial examinations with OCT.

\section{Case Report}

The patient presented herein was a 46-year-old woman who had developed floaters and ocular pain in her left eye 11 days previously. She had no previous ophthalmic history, and her general condition was good. On presentation, best-corrected visual acuity was 20/16 in the right eye and 6/20 in the affected eye, and intraocular pressure was normal. A slit-lamp examination showed keratic precipitates and inflammatory cells $(2+)$ in the anterior chamber of the left eye (fig. 1a). A fundus examination of the left eye revealed vitreous opacities (VO), moderate disc swelling and hyperemia, perivascular retinal hemorrhage, and yellowwhite patches of necrotizing retinal lesions in the superotemporal area (fig. 1b). A fundus examination of the right eye showed no abnormalities. Fluorescein angiography with Optos 200Tx (Optos plc., Dunfermline, UK) showed hyperfluorescence of the optic disc, dye leakage, and a nonperfusion area around the necrotizing lesions (fig. 1c). Spectral-domain (SD)- 
Sogawa et al.: Rare Association of Perivascular Granulomatous Lesions in a Patient with Acute Retinal Necrosis

OCT (Spectralis, Heidelberg Engineering, Heidelberg, Germany) confirmed high-intensity and uniform deposits in the fovea of the left eye (fig. 1d). The retinal layer structure was maintained around these deposits. SD-OCT images revealed several high-intensity granulomatous lesions in the perivascular area (fig. 1e). The retinal pigment epithelium and choroid were intact. A peripheral blood test, including a blood cell count, C-reactive protein level, and serum angiotensin-converting enzyme activity were within normal ranges. The patient did not have HIV; however, polymerase chain reaction of the aqueous humor revealed varicella-zoster virus infection. ARN was diagnosed based on clinical observations and the rapid progression of retinal necrosis.

Intravenous acyclovir (30 mg/kg/day), intravenous betamethasone $(6 \mathrm{mg} /$ day for 3 days) followed by oral prednisone (30 mg/day), and aspirin (100 mg/day) were initiated after the patient was admitted. This treatment was changed to oral valaciclovir $(3,000 \mathrm{mg} / 3$ times a day), and oral prednisone was gradually tapered over 2 months. Previous studies reported that prophylactic laser photocoagulation may help to prevent and reduce the incidence of retinal detachment [7]. Therefore, we performed prophylactic laser photocoagulation on the surrounding necrotizing lesions on day 6 of her admission. Keratic precipitates, anterior chamber cells, and VO improved shortly after starting this treatment. Yellow-white retinal lesions also gradually resolved. Primary granulomatous lesions initially did not respond to the treatment but gradually resolved within 4 weeks.

Serial examinations of granulomatous lesions by SD-OCT revealed that elevated lesions protruding from the inner retina to the vitreous cavity resolved within 6 months (fig. 2a-c). Recurrence was not observed during a 6-month follow-up. OCT images showed that the retinal structure was maintained and retinal thinning did not progress. The appearance of deposits in the fovea was similar to that of granulomatous lesions; however, they did not protrude from the retina. These lesions also responded to the treatment and finally resolved (fig. 2d-f). In the last examination after a 6-month follow-up, visual acuity in the affected eye improved to 14/20 with no evidence of recurrence, retinal detachment, or active inflammation (fig. $3 \mathrm{a}-\mathrm{c}$ ). The patient continues to be monitored untreated without complications.

\section{Discussion}

This case demonstrates the rare association of perivascular granulomatous lesions with $\mathrm{ARN}$, ordinarily manifesting as fulminant inflammation with destruction of the retina. We also showed treatment-associated and sequential changes in perivascular granulomatous lesions with noninvasive SD-OCT examinations. OCT images of retinal granulomatous lesions with ARN have not been reported previously. Therefore, to the best of our knowledge, this is the first patient to show atypical ARN caused by mild inflammation and/or an unknown pathogenesis.

Culbertson et al. [6] previously described the pathology of ARN retinas. The area of the necrotic retina was sharply demarcated from the more preserved area of the adjacent retina. Furthermore, the retinal structure with necrotic lesions was completely destroyed and retinal thinning was observed. Mononuclear lymphocytes with predominantly plasma cell infiltrations were detected in the choroid adjacent to the necrotic retina. Large amounts of viral particles were observed in the inner retinal layers and the transitional zone between necrotic lesions and the preserved retina but not in multiple sections of the vascular endothelium, optic nerve, or choroid [6]. Cytotoxic T cells have been implicated in the pathogenesis of ARN [8]. These inflammatory cells aggravate retinal necrosis and ultimately lead to the destruc- 
Sogawa et al.: Rare Association of Perivascular Granulomatous Lesions in a Patient with Acute Retinal Necrosis

tion of infected retinal cells. A comparison of OCT structural images in our case and those in pathological reports indicated that pathological effects were weaker in our case [6].

OCT images of retinal lesions with ARN have revealed highly reflective areas in the inner layers of the retina in the acute phase and a significant reduction in retinal thickness after the regression of yellowish-white lesions in the retina [5]. Our case showed atypical granulomatous lesions in the perivascular area without destruction of the retinal structure. In contrast, the OCT findings of sarcoidosis have shown perivascular inflammation and the formation of granulomas around the peripheral retinal vein. In sarcoidosis, intraretinal granulomas from the outer nuclear layer to the nerve fiber layer sometimes protrude into the vitreous cavity [9]. Macrophages have also been reported to play a role in the pathogenesis of granuloma formation. Based on the pathogenesis and similarities in OCT images, the formation of granulomas in our case may have been similar to the pathogenesis of sarcoidosis [9]. The formation of granulomas does not occur in lesions when the retinal structure is lost; therefore, tissue damage by viral infection may be mild, or another mechanism such as macrophage involvement, similar to granuloma formation, may be responsible.

OCT findings also showed deposits in the foveal region. It currently remains unclear whether retinal inflammation causes foveal deposits. However, this material may have been derived from retinal debris because the fovea is an avascular zone and the formation of granulomas is typically detected in the perivascular area, similar to sarcoidosis. Serial observations using OCT imaging suggested that foveal deposits clearly formed around the perivascular zone. A previous study reported that inflammatory cells and necrotic retinal debris extended from the retina into the overlying vitreous [6]. The deposit formation in foveal lesions is presumed to be derived from the cells and debris surrounding the vitreous cavity. Mechanisms that have not yet been identified may be involved in the formation of foveal deposits.

To the best of our knowledge, we are the first to report perivascular granulomatous lesions in the retinal layer without complete disruption of the retinal structure in the affected area. These results suggest a new pathology for ARN without fulminant inflammation.

\section{Statement of Ethics}

The Institutional Review Board of the Osaka University Medical School approved the research protocol, and the procedures conformed to the tenets of the Declaration of Helsinki.

\section{Disclosure Statement}

The authors have no proprietary or commercial interest in any material discussed in this paper.

\section{References}

1 Urayama A, Yamada N, Sasaki T, et al: Unilateral acute uveitis with retinal periarteritis and detachment. Jpn J Clin Ophthalmol 1971;25:607-619.

2 Holland GN: Standard diagnosis criteria for the acute retinal necrosis syndrome. Am J Ophthalmol 1994;117:663-667.

-3 Cochrane TF, Silvestri G, McDowell C, Foot B, McAvoy CE: Acute retinal necrosis in the United Kingdom: results of a prospective surveillance study. Eye (Lond) 2012;26:370-377. 
Case Reports in

Ophthalmology

\begin{tabular}{l|l}
\hline Case Rep Ophthalmol 2015;6:373-379 \\
\hline DOI: 10.1159/000442084 & $\begin{array}{l}\text { ○ 2015 The Author(s). Published by S. Karger AG, Basel } \\
\text { www.karger.com/cop }\end{array}$ \\
\hline
\end{tabular}

Sogawa et al:: Rare Association of Perivascular Granulomatous Lesions in a Patient with Acute Retinal Necrosis

4 Sugita S, Shimizu N, Watanabe K, Mizukami M, Morio T, Sugamoto Y, Mochizuki M: Use of multiplex PCR and real-time PCR to detect human herpes virus genome in ocular fluids of patients with uveitis. Br J Ophthalmol 2008;92:928-932.

-5 Suzuki J, Goto H, Minoda H, Iwasaki T, Sakai J, Usui M: Analysis of retinal findings of acute retinal necrosis using optical coherence tomography. Ocul Immunol Inflamm 2006;14:165-170.

$\checkmark 6$ Culbertson WW, Blumenkranz MS, Pepose JS, Stewart JA, Curtin VT: Varicella zoster virus is a cause of the acute retinal necrosis syndrome. Ophthalmology 1986;93:559-569.

7 Meghpara B, Sulkowski G, Kesen MR, Tessler HH, Goldstein DA: Long-term follow-up of acute retinal necrosis. Retina 2010;30:795-800.

-8 Vann VR, Atherton SS: Neural spread of herpes simplex virus after anterior chamber inoculation. Invest Ophthalmol Vis Sci 1991;32:2462-2472.

-9 Wong M, Janowicz M, Tessler HH, Goldstein DA: High-resolution optical coherence tomography of presumed sarcoid retinal granulomas. Retina 2009;29:1545-1546.
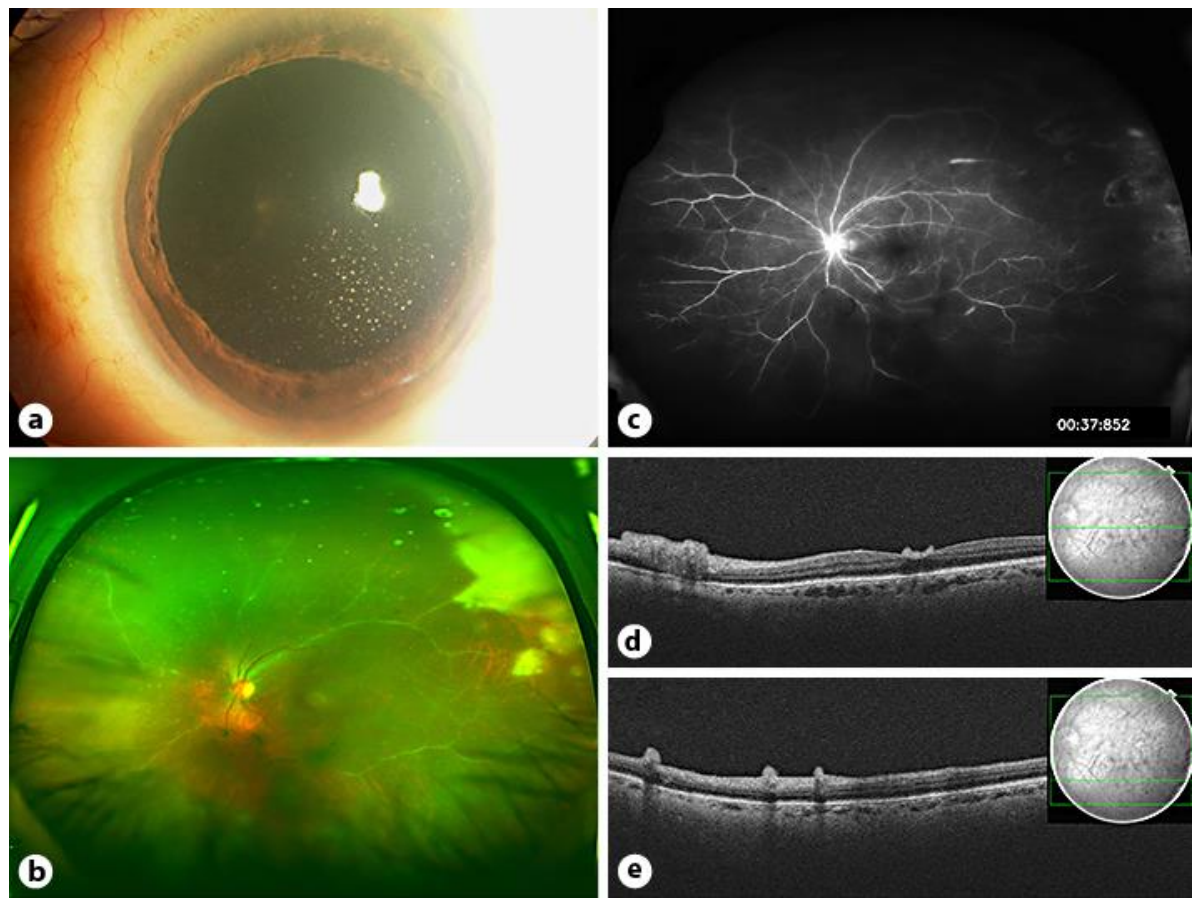

Fig. 1. a Slit-lamp examination showing keratic precipitates and inflammatory cells (2+) in the anterior chamber. $\mathbf{b}$ A fundus photograph showing VO, moderate disc swelling and reddening, perivascular retinal hemorrhage, and yellow-white patches of necrotizing retinal lesions in the superotemporal area at the first visit. c Fluorescein angiography showing hyperfluorescence of the optic disc, dye leakage, and a nonperfusion area around the necrotizing lesions. OCT images showing high-intensity and uniform deposits in the fovea (d) and high-intensity and uniform lesions protruding from the perivascular area (e). 


\section{Case Reports in \\ Ophthalmology}

\begin{tabular}{l|l}
\hline Case Rep Ophthalmol 2015;6:373-379 \\
\hline DOI: 10.1159/000442084 & $\begin{array}{l}\text { @ } 2015 \text { The Author(s). Published by S. Karger AG, Basel } \\
\text { www.karger.com/cop }\end{array}$ \\
\hline
\end{tabular}

Sogawa et al:: Rare Association of Perivascular Granulomatous Lesions in a Patient with Acute Retinal Necrosis

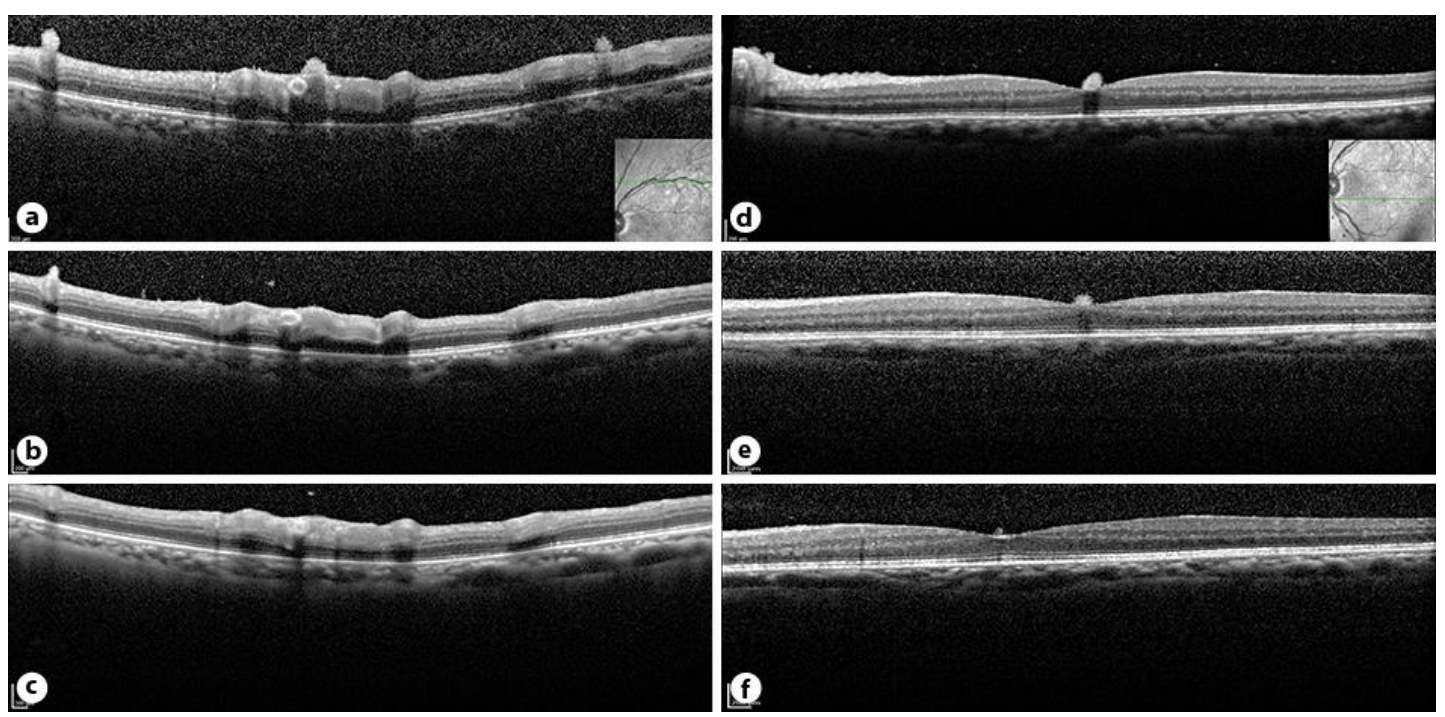

Fig. 2. OCT images of perivascular granulomatous lesions 16 days (a), 30 days (b), and 58 days (c) after treatment initiation. Pedunculated, high-intensity, and uniform lesions that were raised in the perivascular area gradually resolved. OCT images of foveal deposits 16 days (d), 30 days (e), and 58 days (f) after treatment initiation showing gradual resolution. 


\section{Case Reports in \\ Ophthalmology}

\begin{tabular}{l|l}
\hline Case Rep Ophthalmol 2015;6:373-379 \\
\hline DOI: 10.1159/000442084 & $\begin{array}{l}\text { C } 2015 \text { The Author(s). Published by S. Karger AG, Basel } \\
\text { www.karger.com/cop }\end{array}$
\end{tabular}
www.karger.com/cop

Sogawa et al.: Rare Association of Perivascular Granulomatous Lesions in a Patient with Acute Retinal Necrosis
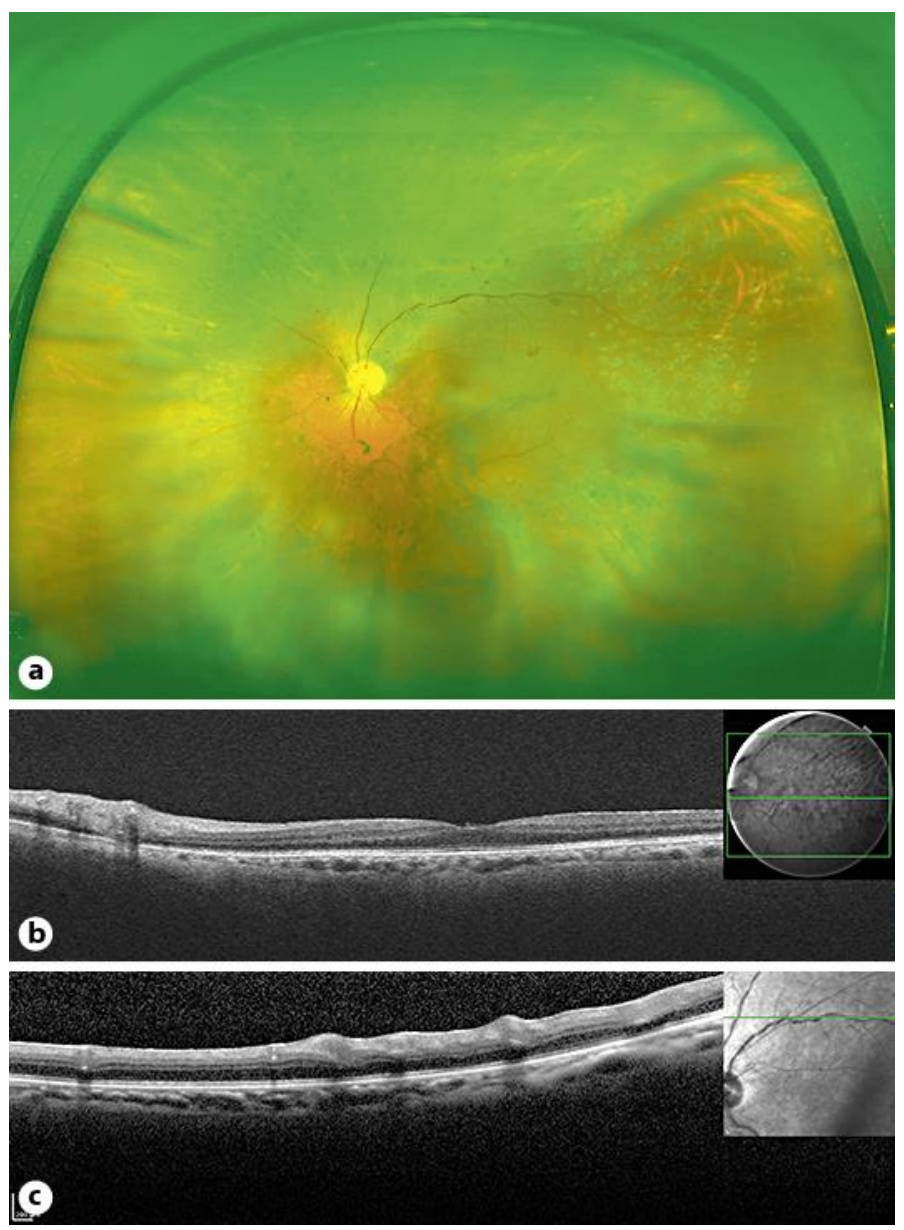

Fig. 3. Five months after the start of treatment. a A fundus photograph showing retinal atrophy in the necrotizing retinal lesions, prophylactic photocoagulation scars, and resolutions of VO. OCT image showing the resolution of foveal deposit (b) and perivascular granulomatous lesions (c) without destruction of the retinal structure. 